\title{
Solar wind density intercomparisons on the WIND spacecraft using WAVES and SWE experiments
}

\author{
M. Maksimovic, ${ }^{1,2}$ J.-L. Bougeret, ${ }^{1}$ C. Perche, ${ }^{1}$ J.T. Steinberg, ${ }^{3}$ \\ A.J. Lazarus, ${ }^{3}$ A.F. Viñas, ${ }^{4}$ and R.J. Fitzenreiter ${ }^{4}$
}

\begin{abstract}
We present solar wind density comparisons using three different instruments on the WIND spacecraft: The thermal noise receiver (TNR) on the WAVES experiment which yields the total electron density from the detection and the analysis of the electron plasma peak; the SWE Faraday cup detectors which measure ion energy per charge spectra and yield the proton and alpha particle densities in the solar wind; and finally the SWE electron spectrometer which provides $3 \mathrm{D}$ electron velocity distribution functions and thus the total electron density. The density comparison between the TNR and the SWE Faraday cups shows a remarkably good agreement, with a systematic offset which is lower than $1 \%$ and fluctuations of up to $\approx 5 \%$ around this value. Due to the well known spacecraft electrical potential effects, the SWE electron spectrometer densities, uncorrected from the potential, exhibit larger differences when compared with the TNR or the SWE Faraday cups measurements. Using these latter densities as reference, we compute a rough estimation of the spacecraft potential.
\end{abstract}

\section{Introduction}

The accurate measurement of the solar wind electron and ion densities is a key element in understanding the physics of the solar wind itself or the physics of the various solarterrestrial links. Several kinds of electrostatic analyzers have been flown in the solar wind, yielding excellent data. Most of these detectors are either curved plate electrostatic analyzers or are gridded detectors such as Faraday cups and retarding potential analysers. However the observations made by these instruments can be altered by various factors such as the spacecraft (s/c) charging effects or the presence of photoelectrons in the case of electron analyzers, or possible temporal variations of the instrument internal calibrations. For instance, systematic discrepencies between ion density measurements taken by different analyzers have been reported by Russell and Petrinec [1992] or Petrinec and Russell [1993], though these descrepencies are a subject of some controversy (see the comment by Paularena and Lazarus [1994] and the reply by Petrinec and Russell [1994]).

In this paper, we present solar wind density comparisons using three different and independent detectors on the WIND s/c: the thermal noise receiver (TNR) on the

\footnotetext{
${ }^{1}$ Observatoire de Paris-Meudon, France

${ }^{2}$ Now at Space Science Dept. of ESA, The Netherlands

${ }^{3}$ Center for Space Research, Massachusetts Institute of Technology, Cambridge, MA

${ }^{4}$ NASA Goddard Space Flight Center, Laboratory for Extraterrestrial Physics, Greenbelt, MD
}

Copyright 1998 by the American Geophysical Union.

Paper number 98GL00843.

0094-8534/98/98GL-00843\$05.00
WAVES experiment and the SWE electron spectrometer (ES) both of which yield the total electron density, and the SWE Faraday cup (FC) detectors which yield the proton and alpha particle densities. The FC and ES give traditional direct measurements of the plasma particles, while the TNR provides a novel method of determining particle density from the electric wave signature. In Section 1, we describe the three experiments. In Section 2, we present the density comparisons and the $s / c$ potential determination.

\section{Experiments}

\section{The WAVES/TNR experiment}

The WAVES/TNR [Bougeret et al., 1995] was mainly designed to measure the electron thermal noise (TN) fluctuations in the solar wind. The analysis of the frequency spectrum of the TN yields both the density and temperature of the solar wind electrons [Meyer-Vernet and Perche, 1989]. We emphasize on the fact that, using this technique, the solar wind electron density $n_{e}$ can be obtained $(i)$ independently on the model chosen to characterize the electron distributions, (ii) with a very high accuracy: to better than $\approx 3 \%$ for most of the cases [Maksimovic et al., 1995] and (iii) without biases due to the s/c electrical potential or photoelectron perturbations which in general affect particle analyzers [Meyer-Vernet et al., 1997; and references therein].

WAVES/TNR, covers an electron density range from $\approx$ 1 to $\approx 500 \mathrm{~cm}^{-3}$ and an electron temperature range from $\approx$ 10 to more than $10^{6} \mathrm{~K}$.

\section{The SWE Faraday cups (FC)}

The Solar Wind Experiment (SWE) includes two Faraday cup (FC) detectors for measuring the energy-per-charge spectra of solar wind protons and alpha particles [Ogilvie et al., 1995]. Under most conditions the FCs provide the number density, thermal speed and bulk flow velocity of the protons and alpha particles separately. Exceptions are as follows. The alpha particle parameters cannot be obtained when: (i) the proton velocity distribution is too broad (when the ratio of the bulk flow speed to the thermal speed is less than about 5), or (ii) their flux is too much smaller than the proton flux (when the alpha-proton number density ratio is less than about 0.005). But these conditions occur only rarely in the solar wind at one AU.

SWE FC ion data used for the comparisons presented here are derived from an analysis algorithm slightly more sophisticated than that used to produce the Key Parameters (KPs, see http://web.mit.edu/space/ www/wind/wind.html). The FC ion data herein were analyzed as follows. Energy-per-charge scans from both FCs are collected for several different $s / c$ azimuth angles and analyzed together. Modeling the proton velocity distribution as a single temperature (isotropic) convecting Maxwellian, 
Table 1. The comparison time periods

\begin{tabular}{cccccc}
\hline yr,mo,day & $\begin{array}{c}V_{S W}(\mathrm{~km} / \mathrm{s}) \\
\text { min-max, ave }\end{array}$ & $\begin{array}{c}V_{S W} / V_{p T h} \\
\min -\max , \text { ave }\end{array}$ & $\begin{array}{c}\text { density }\left(\mathrm{cm}^{-3}\right) \\
\min -\max , \text { ave }\end{array}$ & $\begin{array}{c}H e^{++} / H^{+} \\
\text {ave }\end{array}$ & $\begin{array}{c}\text { Comments on } \\
\text { solar wind conditions }\end{array}$ \\
\hline & & & & & \\
$95,01,18^{*}$ & $350-509,414$ & $5.9-17.6,11.0$ & $3.8-37.7,16.3$ & 0.036 & Onset of a weak stream \\
$95,02,07$ & $350-494,397$ & $6.4-22.3,12.9$ & $2.3-9.7,4.7$ & 0.051 & Streamer belt flow, $V_{S W} \nearrow$ \\
$95,02,08$ & $372-455,415$ & $9.6-27.2,18.0$ & $3.4-23.0,8.7$ & 0.025 & Streamer belt flow, $V_{S W} \searrow$ \\
$95,05,01$ & $354-419,382$ & $8.3-20.5,15.0$ & $4.7-21.4,7.5$ & 0.033 & Streamer belt flow \\
$95,05,22$ & $328-398,366$ & $9.0-18.5,14.6$ & $8.3-22.2,14.7$ & 0.018 & Streamer belt flow \\
$95,05,30$ & $368-740,583$ & $5.0-14.4,8.6$ & $4.2-27.2,9.9$ & 0.039 & Onset of high speed stream
\end{tabular}

*Hours 0-16 only.

the best fit distribution is found using a non-linear least squares method. The fit yields the proton velocity, density, and most probable thermal speed. The resulting fit is used to subtract the proton contribution to the measurements, and the fit procedure is repeated to determine the alpha particle parameters. The densities for protons or alpha particles may be somewhat inaccurate when the distribution functions have strong non-Maxwellian features.

The results from the algorithm used here generally agree quite well with those from the KP algorithm. For very narrow distributions (when the ratio of the bulk flow speed to the most probable thermal speed is larger than 25) the KP thermal speeds may be slightly high and the densities slightly low; the analysis results used in this report will be more accurate.

\section{The SWE electron spectrometer (ES)}

The SWE plasma instrument on the WIND s/c contains also a tri-axial electron spectrometer [Ogilvie et al., 1995] which performs 3-D measurements of the electron distribution functions. There are two sets of three electrostatic analyzers configured in such a way that pairs of detectors look in opposing directions. The field of view of each detector is $8^{\circ} \times 10^{\circ}$. The electron distributions are measured in 16

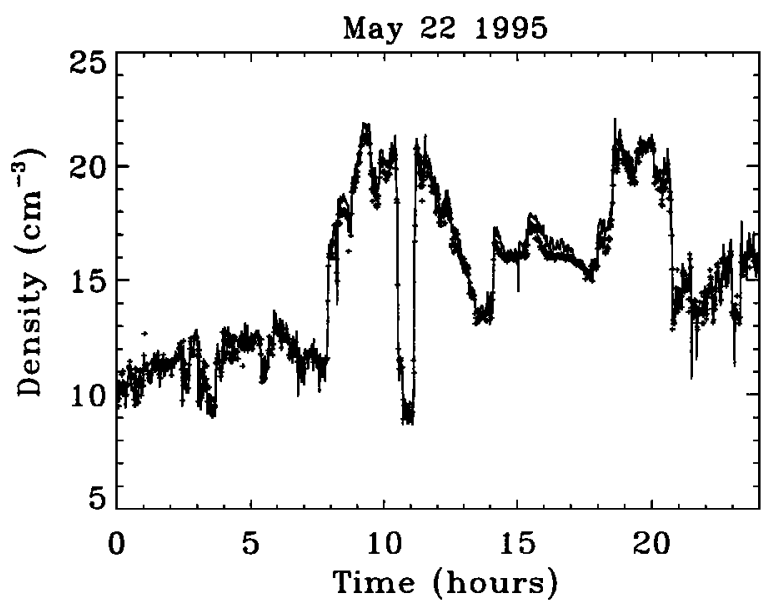

Figure 1. Time variation of the solar wind density as measured by the SWE FC (solid line) and the TNR (crosses), on May 22, 1995. The agreement between the two measurements is very good, even for periods where the density varies a lot during a short time. steps over the energy range from $10 \mathrm{eV}$ to $22 \mathrm{keV}$ in 0.5 sec by the six detectors as the spacecraft rotates through $30^{\circ}$. During one complete $3 \mathrm{sec}$ spacecraft rotation, there are 512 measurements of the distribution function in velocity and configuration space. The electron density, bulk velocity, pressure tensor, and heat flux vector are obtained from velocity moments computed using the measured distribution functions.

\section{Solar wind density comparisons}

\section{The TNR-FC density comparison}

Our TNR-FC comparison is made with the data acquired during six different days in 1995. The list of those days is given in the Table 1 . The periods were selected because they have enough data for the comparison and because they have also a significant temporal variation of the density. The whole data set contains solar wind densities ranging from between 2 and $40 \mathrm{~cm}^{-3}$, as well as a range of solar wind flow conditions including typical slow and fast speeds, narrow and broad ion distributions, and a range of $\mathrm{He}^{++} / \mathrm{H}^{+}$density ratios (see Table 1 ). Thus any sensitivity of the density determination by any of the different methods to differing flow conditions should become apparant.

For the present study we have used the high time resolution data from the two experiments: one measurement every $4.5 \mathrm{sec}$ for TNR and every $90 \mathrm{sec}$ for FC. Since the TNR time resolution is the best, we have used for the comparisons, the FC density measurements at the times they are obtained, and averages of the TNR density measurements over inter-

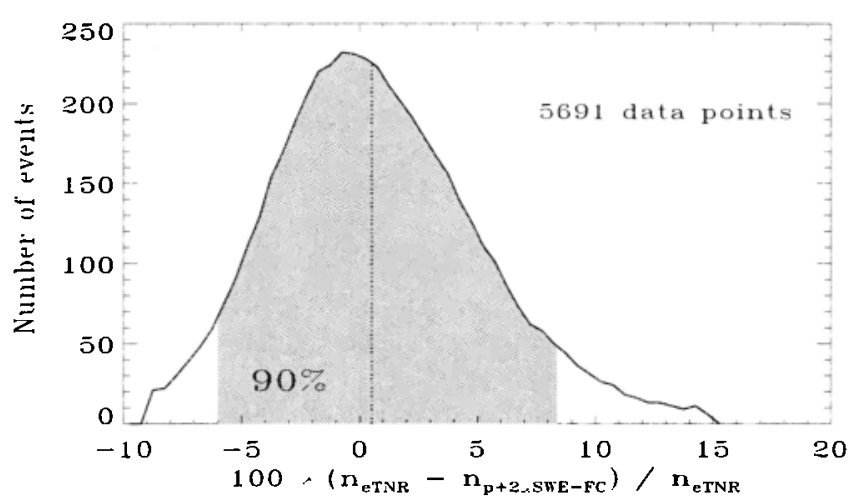

Figure 2. The Histogram of the quantity $\delta_{\mathrm{TNR}-\mathrm{FC}}=100 \mathrm{x}$ $\left(n_{e \mathrm{TNR}}-n_{\mathrm{FC}}\right) / n_{e \mathrm{TNR}}$. See the text for more explanations. 
Table 2. The possible dependence of the TNR-FC density comparison on the solar wind parameters. For each of the data (sub)sets, $\left\langle\delta_{\mathrm{TNR}-\mathrm{FC}}\right\rangle$ represents the median value of $\delta_{\mathrm{TNR}-\mathrm{FC}}$ and $\delta_{1}$ and $\delta_{2}$ are the values within $90 \%$ of the (sub) sets data are contained. $\left\langle\delta_{\mathrm{TNR}-\mathrm{FC}}\right\rangle, \delta_{1}$ and $\delta_{2}$ are very similar in all the cases, indicating that there is no evident dependence of the TNR-FC density comparison on $V_{S W}$ or $T_{p}$.

$\left\langle\delta_{\mathrm{TNR}-\mathrm{FC}}\right\rangle, \delta_{1}$ and $\delta_{2}$
All data set

Data $(44 \%)$ with $T_{p}>410^{4} \mathrm{~K}$

Data (56\%) with $T_{p}<410^{4} \mathrm{~K}$

Data $(38 \%)$ with $V_{S W}>400 \mathrm{~km} / \mathrm{s}$

Data $(62 \%)$ with $V_{S W}<400 \mathrm{~km} / \mathrm{s}$
FC disagreements. However, this is not the case for other periods of disagreement, for which the short time scale fluctuations of the solar wind density or bulk velocity need to be examined more carefully. This exceeds the scope of the present paper.

\section{The ES-FC-TNR density comparisons}

If we now compare the TNR electron density measurements or the FC density measurements with those obtained simultaneously by the ES, the agreement is far from being as good as previously. For instance, the ratios $n_{e E S} / n_{e T N R}$ or $n_{e E S} / n_{\text {FC }}$ range from $\approx 1$ to $\approx 2$. This result is completely expected. It is due to the well known s/c potential effects [Scime et al., 1994]. Since the WIND s/c charges positively, the solar wind electrons are attracted to it and form an electron plasma cloud around the s/c where the electron density is enhanced. Thus the density observed by ES is larger than the true solar wind density. This is not the case neither for the TNR density measurement, since, as already noted in section 1 , the technique involved in that case is immune to the $\mathrm{s} / \mathrm{c}$ potential, nor for the FC density measurement, since the solar wind ions have energies much larger than the typical s/c potential. Hence if we assume that both TNR and FC measure the true solar wind density, the ratios $n_{\text {eES }} / n_{\text {eTNR }}$ or $n_{e E S} / n_{\mathrm{FC}}$ are naturally larger than one.

Actually it is possible to get a rough estimation of the s/c potential $\phi_{s / c}$. In order to do this, one can assume that the velocity distribution observed at the $\mathrm{s} / \mathrm{c}$ is obtained from the true distribution in the solar wind, using Liouville's theorem and the energy conservation: $v^{2}=v^{\prime 2}-2 e \phi_{\mathrm{s} / \mathrm{c}} / m_{e}$, where $v$ is the velocity in the solar wind of an electron that passes through the $\mathrm{s} / \mathrm{c}$ potential and whose observed velocity, when it is collected at the $\mathrm{s} / \mathrm{c}$, is $v^{\prime}$. Under these assumptions, it can be shown that, in a first approximation,

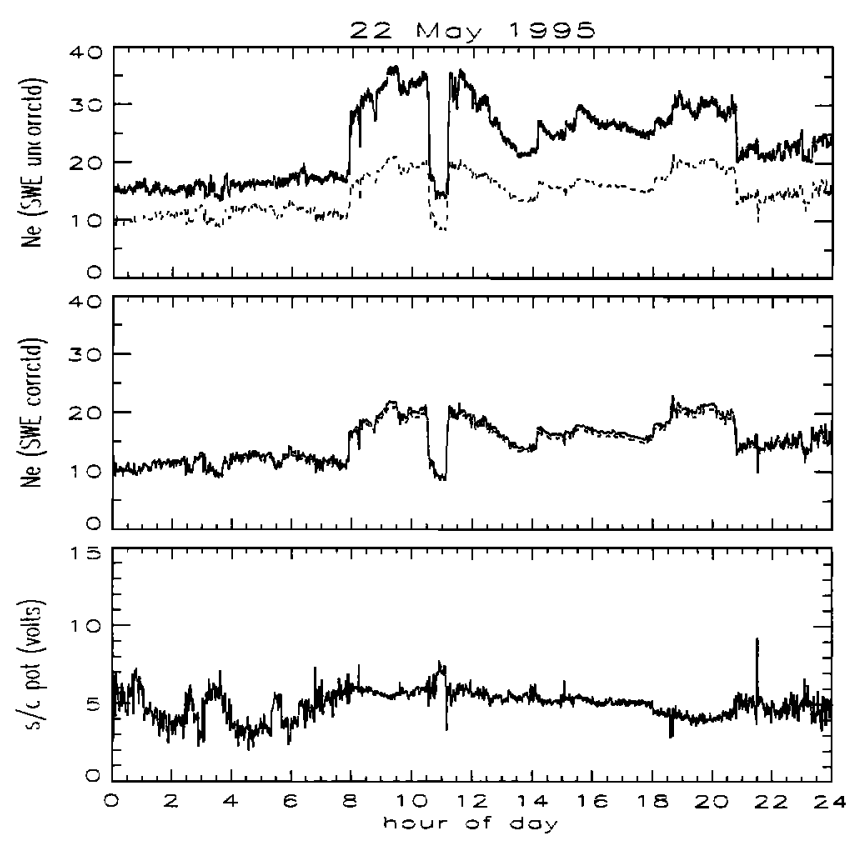

Figure 3. Time variation of the solar wind electron and ion densities as measured by SWE on May 22, 1995. On the lower panel, an estimation of the spacecraft electrical potential. See the text for more explanations. 
the ES electron density, uncorrected from $\phi_{\mathrm{s} / \mathrm{c}}$, is given by: $n_{e \mathrm{ES}} / n_{e}^{*} \approx \exp \left(\phi_{\mathrm{s} / \mathrm{c}} / k T_{e}^{*}\right)(1)$, where $n_{e}^{*}$ and $T_{e}^{*}$ are the true solar wind electron density and temperature. Thus, assuming that TNR and FC measure the true solar wind density $n_{e}^{*}$, one can use this latter expression in order to get a rought estimation of $\phi_{\mathrm{s} / \mathrm{c}}$. This kind of expression has already been used for instance by Scudder et al. [1981] to determine the Voyager s/c potential in the plasma electron environment of Jupiter, using the Voyager observed ion density as the reference density. Note that in expression (1) we of course use the ES electron density measurements uncorrected from the $\mathrm{s} / \mathrm{c}$ potential but from which the photoelectron density has been removed. Note also that we use the electron temperature measured by ES before the s/c potential correction. This mesurement is also altered by the charging effects, but less than for the density.

In Figure 3 we present, for the day 22 May 1995, the spacecraft potential $\phi_{s / c}$ computed from expression (1) and using the FC ion density as reference. In the upper panel of Figure 3, we have displayed both the ES uncorrected electron density (full line) and the FC ion density (dashed line). The ratio between these two densities ranges roughly from 1.3 to 1.7. Using expression (1) we have computed the $\mathrm{s} / \mathrm{c}$ potential which is displayed on the lower panel of Figure 3. $\phi_{\mathrm{s} / \mathrm{c}}$ ranges roughly from 2 to 7 Volts. Having these estimations for the potential, it is possible then to compute the corrected ES electron densities, using again Liouville's theorem in order to correct the electron distributions observed at the s/c. In the middle panel of Figure 3, we have displayed, this time, the corrected ES electron density (full line) and again the FC ion density (dashed line). The discrepancies between the two measurements are now much smaller and the ratio $n_{e E S \text { corrctd }} / n_{F C}$ is now much closer to one.

\section{Concluding remarks}

We have presented solar wind density comparisons using three different instruments on the WIND spacecraft. The density comparison between the Thermal Noise Receiver and the SWE Faraday cups shows a remarkably good agreement, with a systematic difference which is lower than $1 \%$. This is, to our knowledge, the first time that two quite different instruments agree so well in measuring the solar wind density. Nevertheless, some experimental biases, which yield absolute density differences between the two experiments larger than $\approx 5 \%$, have still to be investigated.

We have also determined the spacecraft electrical potential which affects the density measurements by the SWE electron spectrometer. We have shown that a spacecraft potential ranging roughly from 2 to 7 Volts can explain the overestimation made by the SWE Electron Spectrometer when measuring the solar wind electron density.
Acknowledgments. This work was supported mainly by the Centre National d'Etudes Spatiales and partially by a grant from the University of Maryland. The work at MIT was supported in part by NASA under grant NAG5-2839.

\section{References}

Bougeret, J.-L., et al., WAVES: The radio and plasma Wave investigation on the WIND spacecraft, Space Sci. Rev., 71, 231$265,1995$.

Meyer-Vernet, N., and C. Perche, Tool kit for antennae and thermal noise near the plasma frequency, J. Geophys. Res., 94, 2405-2415, 1989.

Meyer-Vernet, N., et al., Measuring plasma parameters with thermal noise spectroscopy, in: Measurements Techniques for Space Plasma, AGU Monograph, eds.: Borovsky J. et al., in press, 1997.

Maksimovic, M., et al., Solar wind electron parameters from quasi-thermal noise spectroscopy and comparison with other measurements on Ulysses, $J$. Geophys. Res., 100, 19,88119,891 .

Ogilvie, K.W., et al., SWE: A comprehensive plasma instrument for the WIND spacecraft, Space Sci. Rev., 71, 55-77, 1995.

Paularena, K.I. and A.J. Lazarus, Comment on "Intercalibration of solar wind instruments during the international magnetospheric study" by S.M. Petrinec and C.T. Russell, J. Geophys. Res., 99, 14,777-14,778, 1994.

Petrinec, S.M. and C.T. Russell, Intercalibration of solar wind instruments during the international magnetospheric study, $J$. Geophys. Res., 98, 18,963-18,970, 1993.

Petrinec, S.M. and C.T. Russell, Reply to comment by K.I. Paularena and A.J. Lazarus, J. Geophys. Res., 99, 14,779$14,780,1994$.

Russell, C.T. and S.M. Petrinec, On the relative intercalibration of the solar wind instruments on IMP-8 and ISEE-3, Geophys. Res. Lett., 19, 961-963, 1992.

Scime, E.E., J.L. Phillips and S.J. Bame, Effects of spacecraft potential on three-dimensional electron measurements in the solar wind, J. Geophys. Res., 99, 14,769-14,776, 1994.

Scudder, J.D., E.C. Sittler Jr. and H.S. Bridge, A survey of the plasma electron environment of Jupiter: A view from Voyager, J. Geophys. Res., 86, 8157-8179, 1981.

J.-L. Bougeret and C. Perche, Département de Recherche Spatiale, CNRS URA 264, Observatoire de Paris, 92195 Meudon Cedex, France.

R.J. Fitzenreiter and A.F. Viñas, Laboratory for Extraterrestrial Physics, NASA Goddard Space Flight Center, Greenbelt, MD 20771.

A.J. Lazarus and J.T. Steinberg, Center for Space Research, Massachusetts Institute of Technology, Cambridge, MA02139.

M. Maksimovic, Solar System Division, Space Science Department of ESA, P.O. Box 299, 2200 AG Noordwijk, The Netherlands. (e-mail: mmaksimo@estec.esa.nl)

(Received December 8, 1997; revised February 10, 1998; accepted February 12, 1998.) 